BULLETIN OF THE

AMERICAN MATHEMATICAL SOCIETY

Volume 80, Number 4, July 1974

\title{
THE EQUICHARACTERISTIC CASE OF SOME HOMOLOGICAL CONJECTURES ON LOCAL RINGS
}

\author{
BY MELVIN HOCHSTER ${ }^{1}$
}

\author{
Communicated by Hyman Bass, December 4, 1973
}

\begin{abstract}
This is an announcement of proofs of the intersection conjecture of Peskine and Szpiro and, hence, also, of $\mathbf{M}$. Auslander's zerodivisor conjecture and of an affirmative answer to Bass' question for any equicharacteristic local ring $R$. The key point is that if $x=x_{1}, \cdots, x_{n}$ is a system of parameters for such an $R$ there exists an $R$-module $M$ (not necessarily of finite type) such that $(x) M \neq M$ and for each $k, 0 \leqq k<n,\left(x_{1}, \cdots, x_{k}\right) M: x_{k+1} R=$ $\left(x_{1}, \cdots, x_{k}\right) M$, i.e. $M$ is a sort of non-Noetherian CohenMacaulay module of depth $n$.
\end{abstract}

0 . The main results. "Ring" means commutative, associative ring with identity, and "local ring" means Noetherian ring with a unique maximal ideal. Unless otherwise indicated $(R, P)$ denotes an $n$-dimensional local ring with maximal ideal $P$, and $x=x_{1}, \cdots, x_{n}$ a system of parameters (henceforth, s.o.p.) for $R$. The first main result is

THEOREM 1. Let $\boldsymbol{x}=x_{1}, \cdots, x_{n}$ be a system of parameters for an equicharacteristic local ring $(R, P)$ of dimension $n$. Then there exists an $R$-module $M$ (not necessarily of finite type) such that $(x) M \neq M$ and for each $k, 0 \leqq k,<n,\left(x_{1}, \cdots, x_{k}\right) M: x_{k+1} R=\left(x_{1}, \cdots, x_{k}\right) M$.

If the conclusion of Theorem 1 holds for $x, M$ we shall say that $M$ is $\boldsymbol{x}$-regular.

The proof of this result is sketched in $\S 1$, and will appear in [H4]. (A preliminary version of [H4], which contains the proof, is available in the Aarhus University Preprint Series.)

In the following corollaries of Theorem 1 the hypothesis that a ring $R$ or $S$ contain a field may be weakened to the hypothesis that each of its local rings, modulo nilpotents, contain a field.

Corollary 1 (intersection CONJeCture of Peskine ANd SZPiro). Let $(R, P)$ be a local ring which contains a field, let $M, N$ be $R$-modules of finite type, and suppose that $\operatorname{Supp}(M \otimes N)=\{P\}$. Then $\operatorname{dim} N \leqq \mathrm{pd}_{R} M$.

AMS (MOS) subject classifications (1970). Primary 13D05, $13 \mathrm{C10.}$

${ }^{1}$ The author began this research while supported by the University of Minnesota and NSF grant GP-37689, and completed it while visiting Aarhus University. 
Here, $\operatorname{dim} N=\operatorname{Krull} \operatorname{dim}\left(R / \operatorname{Ann}_{R} N\right)$, and pd denotes projective dimension.

This was proved (as were the other corollaries) by Peskine and Szpiro [PS] for the case of a field of characteristic $p>0$, for local rings essentially of finite type over any field, and in some other special cases, using local cohomology, the Frobenius functor, and M. Artin's approximation theorem. Our proof of Corollary 1 is new even in characteristic $p$ and does not require local cohomology nor even knowledge of the functor Ext. Corollaries 1 and 2 follow from Proposition 2.2 of [H1] (which uses Theorem 1 of [H3]) and Theorem 1 here.

Corollary 2 (homological KRULl height theOREM). Let $R \rightarrow S$ be $a$ homomorphism of Noetherian rings, $M \neq 0$ an $R$-module of finite type, $I=$ $\mathrm{Ann}_{R} M$, and let $Q$ be a minimal prime of IS. Suppose that $S$ contains a field. Then ht $Q \leqq \operatorname{pd}_{R} M$. (Here, ht $Q=\operatorname{dim} S_{Q}$.)

Corollary 2 is superficially stronger than Corollary 1 , where $N$ may be replaced by $R / J\left(J=\operatorname{Ann}_{R} N\right)$. Let $S=R / J, Q=P / J$, and then Corollary 2 specializes to Corollary 1 .

If $R=Z\left[X_{1}, \cdots, X_{n}\right], M=R /(X) R$, then $\operatorname{pd}_{R} M=n$ and Corollary 2 specializes to the Krull height theorem for $S$.

Corollary 3 (M. Auslander's Zerodivisor CONJECTURE). Let $M \neq 0$ be a module of finite type over a local ring $R$ which contains a field and suppose that $\mathrm{pd}_{R} M$ is finite. If an element of $R$ is not a zerodivisor on $M$, then it is not a zerodivisor on $R$.

Corollary 3 was shown in [PS] to follow from Corollary 1.

COROLlARY 4 (BASS' QUESTION). If $R$ is a local ring which contains a field, and $R$ possesses a nonzero module of finite type and finite injective dimension, then $R$ is Cohen-Macaulay.

The argument in [PS] shows that if a class of local rings is closed under the operations of completion and localization at a prime and Corollary 1 holds for rings in the class, then so does Corollary 4. Equicharacteristic local rings is such a class.

1. Sketch of the proof of Theorem 1. Let $(R, P)$ be any local ring and $\boldsymbol{x}$ an s.o.p. for $R$. If $M$ is an $R$-module, $a \in M$, and $m_{1}, \cdots, m_{k+1} \in M$ are such that $\sum_{i=1}^{k+1} x_{i} m_{i}=0$, let $M^{\prime}=\left(M \oplus R^{k}\right) / R v$, where $v=\left(m_{k+1}, x_{1}, \cdots, x_{k}\right)$, and let $a^{\prime}$ be the image of $a$ under the obvious map $M \rightarrow M^{\prime}$. We call $\left(M^{\prime}, a^{\prime}\right)$ a ( first) modification of $(M, a)$ (of type $k$ ) with respect to $x$. We emphasize that the modification always carries with it a map of pairs, $(M, a) \rightarrow$ $\left(M^{\prime}, a^{\prime}\right)$. If

$$
\left(M_{0}, a_{0}\right) \rightarrow \cdots \rightarrow\left(M_{i}, a_{i}\right) \rightarrow \cdots \rightarrow\left(M_{r}, a_{r}\right)
$$


is a sequence of successive modifications and modification maps of respective types $k_{1}, \cdots, k_{r}$ (we usually omit the phrase "with respect to $\boldsymbol{x}$ " from now on), we shall say that $\left(M_{r}, a_{r}\right)$ is an (rth) modification of $\left(M_{0}, a_{0}\right)$ of type $k=\left(k_{1}, \cdots, k_{r}\right)$. The following lemma is quite easy but very important.

LEMMA 1. Let $\boldsymbol{x}$ be an s.o.p. for a local ring $R$. Then the following two conditions are equivalent:

(1) There exists an $x$-regular module over $R$.

(2) For every $r$, if $(M, a)$ is an rth modification of $(R, 1)$, then $a \notin(x) M$.

Let $X_{1}, \cdots, X_{n}, Y_{1}, \cdots, Y_{q}$ be indeterminates over the integers $Z$, and let $\xi$ denote the system of equations $F_{i}(X, Y)=0,1 \leqq i \leqq h$, where $F_{1}, \cdots, F_{h}$ are elements of $Z[X, Y]$. We shall say that an s.o.p. for a local ring $R$, say $x=x_{1}, \cdots, x_{n}$, is constrained by $\xi$ if the equations $F_{i}(x, Y)=0$, $1 \leqq i \leqq h$, have no common solution for the indeterminates $Y$ in $R$. It is quite easy to see

LEMMA 2. let $n$ and $\boldsymbol{k}=\left(k_{1}, \cdots, k_{r}\right)$ be given, $0 \leqq k_{i}<n, 1 \leqq i \leqq r$. Then there is a system of equations $\xi(n, k)$ (in $n$ indeterminates $X_{1}, \cdots, X_{n}$. and $a$ number of indeterminates $Y_{j}$ which depends on $\left.n, k\right)$ with coefficients in $Z$ such that for every local ring $R$ of dimension $n$ and for every s.o.p. $\boldsymbol{x}=x_{1}, \cdots, x_{n}$ for $R$, the following two conditions are equivalent:

(1) For every rth modification $(M, a)$ of $(R, 1)$ of type $\boldsymbol{k}, a \notin(x) M$.

(2) The s.o.p. $x$ is constrained by $\xi(n, k)$.

This idea was discussed in [H2]. Next, using M. Artin's beautiful approximation theorem [A, Theorem 1.10, p. 26], Chevalley's constructability theorem, the usual devices for descent, and, in the final stages, the theorem of generic flatness, one can show

LEMMA 3. Suppose that a system $\xi$ constrains every s.o.p. of every ndimensional local domain essentially of finite type over a field of positive characteristic. Then it constrains every s.o.p. of every n-dimensional local ring which contains a field of characteristic $O$.

It suffices, by virtue of these lemmas, to prove Theorem 1 for characteristic $p>0$, and one can reduce further to the case where $R$ is a modulefinite extension domain of the formal power series ring $K\left[\left[x_{1}, \cdots, x_{n}\right]\right]$ (char $K=p>0$ ), and the extension of fraction fields is separable. Call an s.o.p. $\boldsymbol{x}$ for $R$ amiable (for $c$ ) if there is an element $c$ of $R$, not nilpotent, such that for all $k, 0 \leqq k<n$, and for all positive integers $t \geqq 1$,

$$
c\left(\left(x_{1}^{t}, \cdots, x_{k}^{t}\right) R: x_{k+1}^{t} R\right) \subset\left(x_{1}^{t}, \cdots, x_{k}^{t}\right) R .
$$


LEMMA 4. If $R$ is a separable module-finite extension domain of a formal power series ring $K\left[\left[x_{1}, \cdots, x_{n}\right]\right]$, then $x_{1}, \cdots, x_{n}$ is an amiable s.o.p. for $R$.

Then Theorem 1 reduces to the following crucial lemma, whose proof is so short that we give it.

Lemma 5. If $R$ is a local ring of positive prime characteristic $p$ and $\boldsymbol{x}$ is an amiable s.o.p. for $R$, then for any rth modification $(M, a)$ of $(R, 1)$, $a \notin(x) M$.

Proof. Suppose $x$ is amiable for $c$, fix $r$, and assume, to the contrary, that $a \in(\boldsymbol{x}) M$. Choose $e$ so that $c^{r} \notin\left(x_{1}^{p^{e}}, \cdots, x_{n}^{p^{e}}\right) R$. Apply the $e$ th iteration of the Frobenius functor (see [PS]) to the sequence of modifications of $(R, 1)$ which produces $(M, a)$. The result is a sequence of modifications of $(R, 1)$ with respect to the s.o.p. $x^{\prime}=x_{1}^{p e}, \cdots, x_{n}^{p^{e}}$ such that, if $\left(M^{\prime}, a^{\prime}\right)$ is the new $r$ th term, $a^{\prime} \in\left(x^{\prime}\right) M^{\prime}$. But from the amiability condition one can easily construct, by induction on $i$, vertical arrows $f_{i}$ in the diagram

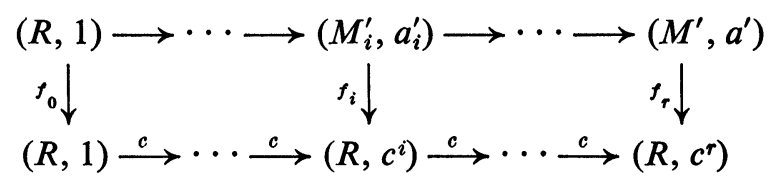

so that it commutes, where $f_{0}=\mathrm{id}_{R}$, and then since $a^{\prime}$ is in $\left(x^{\prime}\right) M, c^{r}$ is in $\left(x^{\prime}\right) R$, a contradiction. Q.E.D.

\section{REFERENCES}

[A] M. Artin, Algebraic approximation of structures over complete local rings, Inst. Hautes Etudes Sci. Publ. Math. No. 36 (1969), 23-58. MR 42 \#3087.

[H1] M. Hochster, Cohen-Macaulay modules, Proc. Kansas Commutative Algebra Conference, Lecture Notes in Math., vol. 311, Springer-Verlag, Berlin and New York, 1973.

[H2] - Constraints on systems of parameters, Proc. Oklahoma Ring Theory Conference, Marcel Dekker, New York, 1974.

[H3] - Grade-sensitive modules and perfect modules, Proc. London Math. Soc. (to appear).

[H4] - Deep local rings, Preprint of preliminary version available in Aarhus University Preprint Series (in preparation).

[PS] C. Peskine and L. Szpiro, Dimension projective finie et cohomologie locale, Inst. Hautes Etudes Sci. Publ. Math. No. 42 (1973), 323-395.

Department of Mathematics, Aarhus University, Aarhus, Denmark

Current address: Department of Mathematics, Purdue University, West Lafayette, Indiana 47907 